\title{
Caracterização demográfica de estagiários em Psicologia no Brasil
}

\author{
Demographic characterization of Psychology interns in Brazil
}

${ }^{[a]}$ Doutoranda em Psicologia Clínica, Instituto de Psicologia, Universidade de São Paulo, São Paulo, SP - Brasil, e-mail: luanflaviabf@gmail.com

${ }^{[b]}$ Doutora em Psiquiatria e Psicologia Médica pela Universidade Federal de São Paulo, professora titular da Pontifícia Universidade Católica do Rio Grande do Sul, Faculdade de Psicologia, Programa de Pós-graduação em Psicologia, Porto Alegre, RS - Brasil, e-mail: marga@pucrs.br

${ }^{[c]}$ Doutora em Psicologia pela Universidade de São Paulo, Instituto de Psicologia, Universidade de São Paulo, São Paulo, SP - Brasil, e-mail marinamonzani@gmail.com

${ }^{[\mathrm{d}]}$ Pós-doutorado em Psicologia Social, pela Universidade de São Paulo, professora da Universidade Federal de São Paulo, Santos, SP - Brasil, e-mail: nancy.unifesp@ gmail.com

${ }^{[\mathrm{e}]}$ Doutor em Psicologia pela Universidade de São Paulo, pós-doutorando em Psicologia Clínica, Instituto de Psicologia, Universidade de São Paulo, São Paulo, SP - Brasil, e-mail: rpereira@usp.br

${ }^{[\mathrm{ff}]}$ Doutora em Psicologia pela Universidade de São Paulo, professora titular do Instituto de Psicologia, Universidade de São Paulo, São Paulo, SP - Brasil, e-mail: efdmsilv@usp.br

\author{
Luan Flávia Barufi Fernandes ${ }^{[a]}$, Margareth da Silva Oliveira ${ }^{[b]}$, Marina Monzani da Rocha ${ }^{[c]}$, Nancy \\ Ramanciotti de Oliveira Monteiro ${ }^{[d]}$, Rodrigo Fernando Pereira ${ }^{[e]}$, Edwiges Ferreira de Mattos \\ Silvares ${ }^{[\mathrm{f}]}$, \& Ana Cláudia Azevedo Peixoto[g]
}

\section{Resumo}

O panorama atual da profissão de psicólogo no Brasil parece estar atravessando um momento de reflexão e questionamento acerca de seu futuro. 0 presente trabalho é um recorte do Projeto Temático Serviços-Escola de Psicologia no Brasil, e seu objetivo é apresentar dados sobre as características demográficas dos alunos de estágios supervisionados dos cursos de graduação em Psicologia. A pesquisa, disponibilizada em um site exclusivo, era composta por um cadastro inicial em que o participante informava dados demográficos: sexo, idade e estado. Participaram da pesquisa 394 alunos. 0 perfil da amostra obtida foi: sexo feminino, idade entre 21 e 30 anos e que cursam faculdades de Psicologia na região Sudeste. Esses dados são similares aos relatados pela literatura nacional acerca do perfil do psicólogo: sexo feminino, com até 34 anos e residentes de regiões metropolitanas, concentradas principalmente na região Sudeste. 0 tamanho da amostra não a faz representativa, mas os dados obtidos forneceram informações importantes sobre quem é este estudante, sua idade e onde estuda. Ademais, estudos de caracterização da população são essenciais para o planejamento de mudanças e aperfeiçoamento do funcionamento de instituições.

Palavras-chave: Serviço-escola. Psicologia. Demografia.

\begin{abstract}
The current scenario of the psychology profession in Brazil seems to be going through a moment of reflection and questioning regarding its future. This paper is a clipping from the Thematic Project of Psychology Services-School in Brazil, and its goal is to present data on the demographic characteristics of students in supervised internships for undergraduate courses in Psychology. The research, available in a dedicated website, consisted of an initial registration in which the participant reported demographic data: gender, age and state. The participants were 394 students. The profile obtained was: female, aged between 21 and 30 and who attend Psychology colleges in the Southeast region. These data are similar to those reported in the national literature on the profile of psychologists: female, aged up to 34 years and residing in metropolitan areas, concentrated mainly in the Southeast region. The sample size is not representative, but the
\end{abstract}

Psicol. Argum. 2014, 32(79), Supl. 2, 19-27 
${ }^{[\mathrm{g}]}$ Doutora em Psicologia pela Universidade Federal do Rio de Janeiro, professora adjunta da Universidade Federal Rural do Rio de Janeiro, Instituto de Educação, Departamento de Psicologia, Rio de Janeiro, RJ - Brasil, e-mail: claudiaapeixoto@gmail.com data provided important information on who is this student, how old is she/he and where she/ he studies. Moreover, population characterization studies are essential for planning changes and improving the operation of institutions.

Keywords: Service-school. Psychology. Demography.

\section{Introdução}

As profissões como sistemas ou estruturas sociais experimentam processos de mudança que alteram continuamente sua relação com a sociedade. No entanto, as profissões diferem no grau em que o processo de profissionalização encontra-se avançado e legitimado pelos pares e pela sociedade (Bastos \& Achcar, 1994; Witter \& Ferreira, 2005).

0 acompanhamento e análise do desenvolvimento de uma profissão devem ser realizados constantemente pelos profissionais e educadores responsáveis pela formação nesta área. Assim como o exercício do psicólogo, enquanto profissão, não poderia ser diferente, pois também sofre estas constantes mudanças e transformações, produtos de sua interação com a sociedade e de sua trajetória histórica (Pereira \& Pereira Neto, 2003).

Segundo Pessotti (1988), em livro promovido pelo Conselho Federal de Psicologia (CFP), a criação dos cursos de bacharelado e licenciatura em Psicologia e a institucionalização da profissão de psicólogo pela Lei 4.119, em agosto de 1962, marcam o início do último de quatro períodos históricos da área no país. Esse atual período da história da Psicologia, o período profissional, foi derivado da criação da lei que promoveu a criação de vários cursos de graduação por todo o Brasil, bem como a criação de cursos de pós-graduação, a organização de entidades de classe e a expansão da atuação do psicólogo em diferentes setores da sociedade.

Após a publicação desse livro, em 1988, outros estudos analisando a profissão e a formação em Psicologia no Brasil foram publicados. Duas outras pesquisas realizadas pelo CFP em 1994 (Achcar, 1994) e em 2001 (Conselho Federal de Psicologia
[CFP], 2001) obtiveram resultados semelhantes aos de 1988, ou seja, a profissão de psicólogo continuava sendo feminina, jovem e localizada nas regiões Sudeste e Sul do Brasil.

No que tange a área de atuação, Bastos e Achcar (1994) afirmam que pesquisas de âmbito nacional, regional e local registraram que a atuação do profissional de Psicologia no Brasil é caracterizada pelo domínio de um modelo restrito e tradicional, que pouco se modificou ao interagir com a sociedade em diferentes contextos. Tal modelo se refere ao atendimento clínico, individual e inspirado no modelo médico de intervenção, o qual é realizado em consultórios particulares e é reproduzido em escolas, hospitais e empresas. Em 2001 (CFP, 2001), a principal área de atuação do psicólogo continua sendo a área clínica.

Em 2009, o CFP elaborou um perfil do psicólogo brasileiro com base nas informações dos psicólogos cadastrados nesta entidade. Eram então 236.100 profissionais, sendo $83,3 \%$ do sexo feminino. Nos Conselhos Regionais de Psicologia (CRPs), a maior porcentagem de mulheres foi $89,4 \%$ (CRP 02-Pernambuco), e a menor, 75,8\% (CRP 15-Alagoas). Em pesquisa pioneira realizada pelo CFP em 1988, 86,6\% dos psicólogos eram do sexo feminino (CFP, 1988). De acordo com os dados de 2009, o percentual de homens elevou-se um pouco nesses vinte anos (de 13,4\% para 16,7\%), mas não a ponto de abalar a predominância feminina nesta atividade ocupacional (Bastos, Gondim \& Rodrigues, 2010).

Witter, Gonçalves, Witter, Yukimitsu e Napolitano (2005) ressaltam que a profissão de psicólogo, como qualquer outra, também foi sensível às transformações sociais, econômicas, políticas e tecnológicas 
que modificaram o contexto da sociedade brasileira ao longo dos anos. Novas formas de atuação foram sendo construídas e testadas, antigas práticas receberam novas roupagens e novas clientelas, e novos contextos foram explorados. Entretanto, estas modificações ainda não eram, segundo os autores, tão visíveis e significativas a ponto de aparecem nas estatísticas.

Pfromm Netto (2006) afirma que um dos entraves para a consolidação da Psicologia como uma ciência e profissão multifacetada e reflexiva às mudanças sociais é a formação profissional que é oferecida desde sua fundação como curso superior.

A formação do psicólogo tem sido objeto de atenção dos estudiosos antes mesmo da existência legal da profissão. Em 1962, a partir da regulamentação do curso superior em Psicologia, vários temas como formação básica e especializada, formação de profissional e/ou cientista, o estágio supervisionado e cursos de pós-graduação foram alvo de debates teóricos e encontros presenciais entre os profissionais da área. Tais temas continuam a ser discutidos e analisados até hoje (Yamamoto, Souza, Silva, \& Zanelli, 2010).

O primeiro currículo mínimo oficial, fixado pelo Conselho Federal de Educação, em 1963, expressava uma visão daquilo que se ensinava e da prática profissional em Psicologia exercida na época. Com a regulamentação do currículo mínimo, observa-se um crescimento considerável da oferta de cursos de Psicologia no Brasil (Lisboa \& Barbosa, 2009).

A década de 1970 do é marcada pelo aumento do número de profissionais formados em Psicologia, pela proliferação dos cursos universitários particulares e pelo aumento da demanda da população por serviços psicológicos. Na década de 1980, nos cursos de graduação em Psicologia, notam-se pequenas mudanças e ajustes individuais nos currículos sem qualquer expressão significativa na formação como um todo. Durante estas duas décadas, muitas áreas do conhecimento refizeram o currículo de seus cursos de formação no Brasil. No entanto, a Psicologia não conseguiu ir além das discussões e dessas sutis mudanças (Witter \& Ferreira, 2005, Lisboa \& Barbosa, 2009).

Na década de 1990 assiste-se a uma considerável mobilização das entidades profissionais da Psicologia, com destaque para o CFP, para debater e concretizar mudanças relacionadas à formação do psicólogo. Em 1996 foi promulgada a Lei de Diretrizes e Bases da Educação Nacional, e o Ministério da Educação instituiu a Comissão de Especialistas em Ensino de Psicologia para criar um projeto de diretrizes curriculares, substituindo o currículo mínimo (Lisboa \& Barbosa, 2009).

De acordo com Witter e Ferreira (2005), após a constituição desta comissão, o CFP e os CRPs organizaram campanhas, reuniões, debates e encontros. Tais mobilizações culminaram com a aprovação das Diretrizes Curriculares para os cursos de Psicologia em 2004 (Brasil, 2004). Como consequências destas novas diretrizes curriculares, os cursos de Psicologia ganharam flexibilidade e a possibilidade de diversificação dos currículos, tendo condições de assegurar uma formação básica, científica e técnica, podendo modificar as atividades acadêmicas para adequá-las às necessidades e às características socioeconômicas e culturais da região em que se situam (Löhr \& Silvares, 2006); um avanço importante para a discussão acerca da formação do psicólogo.

Estas determinações quanto ao estágio supervisionado estão ligadas intimamente a outra determinação das diretrizes curriculares: a previsão da instalação de um Serviço de Psicologia, cuja função é responder às exigências para a formação do psicólogo, congruente com as competências que o curso objetiva desenvolver no aluno e com as demandas de serviço psicológico da comunidade na qual está inserido. Tais Serviços de Psicologia Aplicada são espaços disponibilizados pelas faculdades de Psicologia desde a instituição do currículo mínimo. Um dos aspectos mais discutidos sobre a formação prática do psicólogo é que estes serviços-escola, ou comumente denominados de clínicas-escola, oferecem estágios voltados predominantemente para a clínica, sem propiciar preparação adequada para as demais possibilidades profissionais do psicólogo. Com as novas diretrizes curriculares, há uma esperança e certa cobrança dos pesquisadores e entidades para que estes serviços-escola se reestruturem e ofereçam estágios supervisionados mais coerentes com a configuração atual da sociedade e do mercado de trabalho (Witter et al., 2005).

O panorama atual da profissão de psicólogo no Brasil parece estar atravessando um momento de reflexão e questionamento acerca de seu futuro, principalmente no que se refere à formação e consolidação da Psicologia como ciência. É importante ressaltar que as questões envolvendo a formação

Psicol. Argum. 2014, 32(79), Supl. 2, 19-27 
profissional do psicólogo no Brasil apresentam sustentação de ordem prática e política, mas não científica. Embora haja preocupação com a formação do psicólogo e muitas discussões que ampliaram e consolidaram o conhecimento acerca desta temática, há poucos dados científicos apoiando este corpo de conhecimento (Witter \& Ferreira, 2005).

Com o intuito de contribuir e enriquecer o corpo de conhecimentos (com dados empíricos) sobre a formação do psicólogo no Brasil, foi realizado o Projeto Temático Serviços-Escola de Psicologia no Brasil $^{1}$, cujo objetivo era caracterizar os serviços-escola brasileiros de Psicologia em termos de serviços prestado à clientela, do perfil sociodemográfico dos estagiários e supervisores.

0 presente trabalho é um recorte desse projeto temático. Seu objetivo é apresentar os dados sobre as características demográficas dos alunos de estágios supervisionados dos cursos de graduação em Psicologia.

\section{Método}

Amostra

Participaram da pesquisa 394 alunos de estágios supervisionados em Psicologia de vários estados do Brasil. Os critérios de inclusão foram: ser estudante de graduação em Psicologia e estar cursando algum tipo de estágio supervisionado em Psicologia. 0 método de escolha da amostra foi o de conveniência.

\section{Instrumentos}

A pesquisa foi disponibilizada on-line, em um site exclusivo, e era composta por um cadastroinicial, no qual o participante informava dados demográficos como sexo, idade e estado. (Os resultados destes dados serão apresentados e analisados no

\footnotetext{
${ }^{1}$ O Projeto Temático Serviços-Escola de Psicologia no Brasil foi coordenado pela professora Edwiges Ferreira de Mattos Silvares e faz parte das ações preconizadas pelo grupo de discussão do tema na Associação de PósGraduação e Pesquisa (ANPEPP), tendo recebido apoio do CNPq.
}

presente artigo.) 0 instrumento utilizado para investigar o processo de supervisão recebida durante a realização dos estágios supervisionados pelo aluno de Psicologia foi o "Inventário de levantamento de atividades de supervisão". Este inventário foi criado por Nigan, Cameron e Leverette (1997) e é constituído de 33 questões cujas respostas são baseadas em uma escala Likert de cinco pontos e 7 questões abertas para análise da percepção do estudante quanto à atuação de seu supervisor. Os dados referentes às respostas não serão descritos e analisados no presente artigo, mas serão apresentados em outras publicações.

Ressalta-se que os informantes poderiam requerer, por e-mail, orientações adicionais dos pesquisadores para o preenchimento da pesquisa.

\section{Procedimento}

O contato com os alunos-estagiários ocorreu através de uma carta-convite desenvolvida e padronizada pelo Projeto Temático Serviços-Escola de Psicologia no Brasil. Esta carta foi enviada no início de 2009 aos gestores dos serviços-escola e/ ou coordenadores de curso das instituições de ensino superior de Psicologia em todo o Brasil, a fim de que estes comunicassem aos supervisores e alunos os objetivos da pesquisa e os convidassem a participar. Por opção de alguns gestores, os próprios gestores e/ou supervisores encaminharam os contatos de e-mail dos alunos para os pesquisadores, e estes enviavam o convite para participação na pesquisa diretamente aos alunos.

0 site da pesquisa foi disponibilizado aos participantes em outubro de 2008 e o período de coleta se estendeu até abril de 2010.

Para ampliar a divulgação da pesquisa para além da carta convite, os pesquisadores compareceram a congressos de Psicologia, nos quais stands de divulgação eram montados e computadores conectados à internet eram disponibilizados aos alunos. Houve divulgação da pesquisa em páginas de instituições de ensino superior na internet, bem como por meio de cartazes disponibilizados em várias faculdades de psicologia do Brasil.

A participação do aluno na pesquisa compreendeu o preenchimento no site de cadastro inicial e do "Inventário de levantamento de atividades de supervisão" sobre sua relação com o supervisor durante o desenvolvimento do estágio. 
Análise de dados

Os dados da pesquisa foram coletados e analisados de forma coletiva, preservando a confidencialidade das informações e identidade de cada participante.

Os dados obtidos foram tratados com o auxílio do software estatístico SPSS (Statistical Package for Social Science), versão 19 para Windows, comercializado pela IBM (International Business Machines).

\section{Resultados e Discussão}

Antes de propriamente analisar os resultados do perfil dos estagiários, ressalta-se que uma das dificuldades na realização do estudo foi o preenchimento parcial do cadastro inicial e do questionário pelos participantes. As questões do cadastro inicial apresentaram uma maior taxa de resposta, seguidas pelas questões de múltiplas escolhas e, por último, estão as questões dissertativas referentes ao inventário.

0 total de participantes foi de 402 alunos. Destes, 80 não responderam a nenhuma questão do inventário e 394 preencheram apenas o cadastro inicial.

A amostra total de alunos estagiários de Psicologia participantes da pesquisa é um número pequeno quando comparado ao universo de estudantes dos cursos superiores em Psicologia no Brasil. Segundo o último censo do ensino superior desenvolvido pelo Instituto Nacional de Estudos e Pesquisas Educacionais Anísio Teixeira (INEP, 2011), com base no Cadastro das Instituições de Ensino Superior do Ministério da Educação (MEC), tendo o ano de 2010 como referência, há no Brasil 538 instituições de ensino superior (IESs) em Psicologia.

As informações disponíveis sobre as IESs em psicologia no censo de 2011 estão descritas na Tabela 1.
Assim, pode-se afirmar que o numero de estagiários participantes da pesquisa não é representativo do contingente de alunos matriculados nos cursos de Psicologia no país, uma característica comum a muitas pesquisas anteriores a esta de natureza similar.

Tal dificuldade também foi encontrada, por exemplo, por Witter et al. (2005) ao estudar os currículos, as mudanças curriculares e os estágios oferecidos pelas IESs de Psicologia com o objetivo de estabelecer um levantamento geral da formação e supervisão acadêmica em Psicologia no Brasil. De acordo com os autores, questionários foram remetidos pelo correio às 101 IESs de Psicologia que existiam no Brasil no início da década de 1990 e somente 42 instituições deram retorno, sendo que algumas não responderam a todas as questões solicitadas.

Ressalta-se ainda que, mesmo assim, 402 participantes é uma quantidade considerável de respondentes e permite obter dados significativos sobre as características demográficas dos alunos estagiários de Psicologia e sobre a qualidade das supervisões ofertadas nas IESs.

Outra informação relevante que corrobora a importância de estudos acerca da caracterização dos cursos de graduação em Psicologia, no que se refere à população estudantil e à formação acadêmica, é a escassez de publicações com esta temática na literatura nacional.

Em uma pesquisa bibliográfica realizada em maio de 2012 no site da Biblioteca Virtual em Saúde (BVS), com o uso de combinações com as palavras-chave "estagiários de psicologia”, "caracterização" e "demografia", não foi encontrado nenhum tipo de resultado. Com as palavras-chave "estagiários de Psicologia" (sem combinações) foram localizadas 33 ocorrências, sendo que nenhuma delas se referia a estudos de caracterização desses estagiários, a clínicas-escola ou mesmo a análises sobre a percepção do supervisor. A maior parte deles se referia a

Tabela 1 - Números e porcentagem sobre as instituições de ensino superior em Psicologia fornecidos pelo Censo do Ensino Superior no Brasil de 2011.

\begin{tabular}{lccccccc}
\hline & \multicolumn{2}{c}{ Pública } & \multicolumn{2}{c}{ Privada } & \multicolumn{3}{c}{ Total } \\
\cline { 2 - 8 } & $\mathbf{N}$ & $\%$ & $\mathbf{N}$ & $\%$ & $\mathbf{N}$ & $\%$ \\
Cursos de Graduação presenciais em Psicologia & 110 & 20,4 & 428 & 19,6 & 538 & 100 \\
Matrículas realizadas & 20.611 & 15,1 & 115.809 & 84,9 & 136.420 & 100 \\
Concluintes dos cursos de graduação em Psicologia & 3.042 & 16,6 & 15.269 & 83,4 & 18.311 & 100 \\
\hline
\end{tabular}

Psicol. Argum. 2014, 32(79), Supl. 2, 19-27 
estudos de caso, relatos de experiências realizadas em diferentes áreas de estágio ou estudos de diferentes intervenções.

Deste modo, da amostra total de 402 participantes, para a análise dos dados demográficos foram considerados participantes aqueles indivíduos que responderam as questões do cadastro inicial, ou seja, 394 alunos. Os dados em branco foram desconsiderados nas análises específicas de cada item do cadastro inicial, porém cada resposta preenchida foi incluída, independentemente do grau de preenchimento do estagiário em questão.

Quanto ao sexo, entre os 394 participantes, 82 $(20,8 \%)$ não responderam a este item do cadastro inicial. Os respondentes foram 285 alunos, dos quais $263(66,7 \%)$ eram do sexo feminino e $49(12,5 \%)$ do sexo masculino (Figura 1).

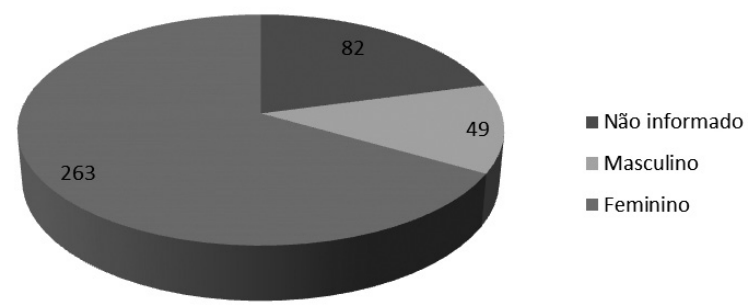

Figura 1 - Sexo dos alunos estagiários de psicologia participantes da pesquisa Projeto Temático Serviços-Escola de Psicologia no Brasil.

Mais da metade dos alunos participantes (66, $7 \%$ ) eram do sexo feminino. Esta predominância das mulheres é uma das características marcantes da profissão de psicólogo no Brasil.

A média de idade desta amostra foi de 25,7 anos, com intervalo de confiança de $95 \%$ de que a média populacional encontra-se entre 24,84 a 26,56 anos e desvio-padrão de 7,7 anos. A idade mínima dos respondentes foi de 18 anos e a máxima de 61 anos.

A distribuição da idade dos participantes por faixa etária está descrita na Tabela 2.

Destaca-se que 60,9\% (n=190) dos estagiários apresentaram idade entre 21 e 24 anos, portanto a grande maioria é composta por indivíduos jovens que ingressaram recentemente na idade adulta. 0 ingresso na faculdade de jovens em torno dos 20 anos é uma condição esperada e estimulada em culturas ocidentais e os dados obtidos sinalizam que
Tabela 2 - Número e porcentagem da idade dos alunos estagiários de psicologia participantes da pesquisa Projeto Temático Serviços-Escola de Psicologia no Brasil por faixa etária.

\begin{tabular}{lcc}
\hline Faixa etária & $\mathbf{N}$ & $\%$ \\
\hline 18 a 20 anos & 28 & 9 \\
21 a 30 anos & 238 & 76,4 \\
\hline 1 a 40 anos & 25 & 8 \\
41 a 50 anos & 12 & 3,8 \\
51 a 61 anos & 9 & 2,8 \\
Total & 312 & 100 \\
\hline
\end{tabular}

alguns jovens brasileiros estão conseguindo cumprir esta expectativa cultural. Aproximadamente $15 \%$ dos estudantes apresentam idade acima de 30 anos e representam adultos que desejam ter diploma de curso superior ou continuam estudando para se preparar melhor para o mercado de trabalho.

A título de curiosidade, na pesquisa conduzida pelo CFP em 1988, a média de idade dos psicólogos foi de 33,6 anos, sendo que $90 \%$ da amostra apresentavam idade entre 22 e 39 anos (CFP, 1988). Na pesquisa de 2009, a média de idade foi de 36,7 anos (desvio-padrão de 10,1 anos), sendo que $25 \%$ da amostra possuíam até 28 anos de idade e 50\% tinham até 34 anos (Bastos, Gondim \& Rodrigues, 2010).

Quanto à amostra dos alunos estagiários de Psicologia, apesar de os dados terem sido coletados com procedimentos e amostragem diferentes das pesquisas realizadas pelo CFP, pode-se observar que há uma tendência de que a profissão continue sendo executada por adultos jovens, visto que $85 \%$ dos respondentes possuem até 30 anos de idade.

Os dados sobre sexo e idade dos alunos estagiários de Psicologia parecem refletir e ser consoantes com o perfil do psicólogo brasileiro obtido nas pesquisas promovidas pelo CFP em 1988 e 2009 (Bastos, Gondim, \& Rodrigues, 2010).Tal perfil indica que a profissão é exercida no Brasil por mulheres jovens e a grande maioria da amostra obtida no presente estudo indica que o estudante de graduação de psicologia é do sexo feminino e tem idade entre 21 e 30 anos.

A pesquisa atingiu 22 unidades da federação, embora muitas tenham tido uma participação pouco expressiva. Oitenta e três participantes $(21,6 \%)$ não responderam a este item do cadastro inicial. As informações acerca do estado brasileiro no qual o aluno estagiário cursa a faculdade de Psicologia estão apresentadas na Tabela 3. 
Tabela 3 - Número e porcentagens dos alunos estagiários que cursam faculdade de Psicologia em cada Unidade Federativa do Brasil. Dados obtidos na pesquisa Projeto Temático Servicos-Escola de Psicologia no Brasil.

\begin{tabular}{lcc}
\hline Estado & N & \% \\
\hline Não Informado & 83 & 21,6 \\
\hline SP (São Paulo) & 87 & 22,6 \\
\hline GO (Goiás) & 48 & 12,5 \\
\hline SC (Santa Catarina) & 33 & 8,6 \\
\hline PR (Paraná) & 28 & 7,3 \\
\hline DF (Distrito Federal) & 20 & 5,2 \\
\hline BA (Bahia) & 17 & 4,4 \\
\hline MG (Minas Gerais) & 16 & 4,2 \\
\hline RJ (Rio de Janeiro) & 16 & 4,2 \\
\hline MT (Mato Grosso) & 6 & 1,6 \\
\hline AM (Amazonas) & 6 & 1,6 \\
\hline PA (Pará) & 5 & 1,3 \\
RN (Rio Grande do Norte) & 5 & 1,3 \\
\hline RO (Rondônia) & 3 & 0,8 \\
\hline RR (Roraima) & 2 & 0,5 \\
\hline PE (Pernambuco) & 2 & 0,5 \\
\hline RS (Rio Grande do Sul) & 2 & 0,5 \\
\hline AP (Amapá) & 1 & 0,3 \\
\hline ES (Espírito Santo) & 1 & 0,3 \\
\hline MS (Mato Grosso do Sul) & 1 & 0,3 \\
\hline PI (Piauí) & 1 & 0,3 \\
\hline SE (Sergipe) & 1 & 0,3 \\
\hline TO (Tocantins) & 0,3 \\
\hline Total & 100 \\
\hline
\end{tabular}

Os estados com maior índice de participação foram São Paulo, Goiás e Santa Catarina. Os estados com menor participação foram Amapá, Espírito Santo, Mato Grosso do Sul, Piauí, Sergipe e Tocantins, com apenas um participante cada. A variação no número de respondentes não se deve apenas ao número de cursos e estudantes em cada local, mas também à divulgação da pesquisa, que foi mais intensa em algumas unidades da federação.

Ao reunir a unidade federativa dos cursos de graduação em Psicologia que tiveram alunos estagiários participantes da presente pesquisa por região geográfica, obtém-se a seguinte configuração (Tabela 4).

O maior número de participantes estudava em IESs da região Sudeste, seguidos pelos estudantes da região Sul. A região Norte foi a que teve menos respostas, pois apenas 18 alunos estagiários colaboraram. Esta configuração da localidade de estudo dos participantes por região geográfica permite
Tabela 4 - Distribuição geográfica das Instituições de Ensino Superior em Psicologia dos alunos participantes da pesquisa Projeto Temático Serviços-Escola de Psicologia no Brasil por região geográfica.

\begin{tabular}{ccc}
\hline Região geográfica & $\mathbf{N}$ & $\%$ \\
\hline Sudeste & 121 & 31,3 \\
Sul & 63 & 16,4 \\
\hline Centro-oeste & 75 & 19,6 \\
\hline Nordeste & 26 & 6,8 \\
\hline Norte & 18 & 4,8 \\
\hline Brasil (Total) & 385 & 100 \\
\hline
\end{tabular}

observar que a região Sudeste foi a mais representada, apesar de, entre as unidades federativas com maior participação, apenas o estado de São Paulo (87 participantes) pertencer a ela. Enquanto o estado de Goiás foi o segundo em número de participantes, a região Centro-Oeste ficou em terceiro lugar. Os estados de Santa Catarina e Paraná colaboraram para que a região Sul fosse a segunda em número de participantes.

É interessante fazer um paralelo desta distribuição geográfica da localidade de estudo dos alunos participantes com a distribuição geográfica dos cursos de psicologia no Brasil. Lisboa e Barbosa (2009) apresentam um perfil dos cursos de graduação em Psicologia no Brasil. Apesar de a coleta de dados ter sido efetuada no ano de 2007 e os dados não serem tão atuais, os autores descrevem a distribuição das faculdades de Psicologia no país por unidade da federação e pela região geográfica. As informações acerca da distribuição das IESs nas regiões geográficas brasileiras estão reproduzidas na Tabela 5.

Tabela 5 - Distribuição geográfica dos cursos de Graduação em Psicologia no Brasil em 2007, segundo Barbosa e Lisboa (2009).

\begin{tabular}{lcc}
\hline Região Geográfica & N & $\%$ \\
\hline Sudeste & 193 & 48,74 \\
Sul & 86 & 21,72 \\
Nordeste & 61 & 15,4 \\
Centro-Oeste & 29 & 7,32 \\
Norte & 27 & 6,82 \\
Brasil (Total) & 396 & 100
\end{tabular}

De acordo com Lisboa e Barbosa (2009), o maior número de IESs que oferecem cursos de Psicologia no Brasil encontra-se na região Sudeste, principalmente em São Paulo (104), estado com maior

Psicol. Argum. 2014, 32(79), Supl. 2, 19-27 
participação de respondentes da pesquisa e local com provável maior divulgação do estudo, sendo ainda a localidade onde residia a grande parte dos pesquisadores.

Observa-se que as regiões com maior número de alunos participantes na pesquisa - Sudeste e Sul - são também as regiões que mais oferecem cursos de Psicologia. A região Norte é a que menos possui IESs em psicologia, assim como foi a que menos teve alunos estagiários que colaboraram com o estudo. A única divergência nos dados encontra-se na terceira e quarta região, nas quais a ordem se inverte na distribuição geográfica: enquanto em número de participantes a região Centro-Oeste ficou em terceiro, na oferta de IESs em Psicologia esta região está em quarto lugar, atrás da região Nordeste, que possui o dobro de faculdades de Psicologia. Esse dado sugere a possibilidade de maior divulgação da pesquisa nessa região, principalmente a partir de comunicações em congressos.

De modo geral, parece que a participação dos alunos estagiários de Psicologia na pesquisa foi coerente com as áreas de maior concentração da localização dos cursos de graduação em Psicologia oferecidos no país.

É importante ressaltar ainda outra limitação do estudo: uma vez que os questionários foram respondidos on-line, sem nenhum tipo de verificação de identidade, não há garantias absolutas da correspondência dos participantes inscritos com os critérios de inclusão do trabalho. Essa verificação só foi realizada quando a coleta foi feita presencialmente nos eventos científicos de Psicologia em que se divulgou a pesquisa.

\section{Conclusões}

Este trabalho é um recorte da pesquisa Projeto Temático Serviços-Escola de Psicologia no Brasil, a qual pretendia caracterizar os serviços-escola de Psicologia no país em termos de funcionamento, estrutura e qualidade das supervisões e traçar um perfil dos alunos e supervisores dos estágios ofertados em Psicologia. 0 presente artigo teve como objetivo descrever e analisar os dados demográficos dos alunos estagiários em Psicologia que acessaram o site da pesquisa e responderam o questionário on-line.
Foram participantes desta análise específica 394 alunos, cujo perfil definido foi: sexo feminino, idade entre 21 e 30 anos e que cursam faculdades de Psicologia na região Sudeste, principalmente no estado de São Paulo. Os dados obtidos são similares aos relatados pela literatura nacional acerca do perfil do psicólogo: profissão exercida predominantemente pelo sexo feminino, com idade até 34 anos e residentes de regiões metropolitanas, concentradas principalmente na região Sudeste. Tal similaridade de perfis sugere uma possível manutenção das características marcantes da profissão de psicólogo no Brasil: grupo ocupacional essencialmente feminino e jovem.

De acordo com o mais recente Censo do Ensino Superior no Brasil (INEP, 2011), em 2010 havia 538 cursos de ensino superior em Psicologia no país, sendo que, neste ano, 136.420 matrículas de novos estudantes em psicologia foram realizadas, e 18.311 estudantes se tornaram psicólogos. Estes dados indicam um constante crescimento da profissão no Brasil.

\section{Considerações Finais}

Estudantes de Psicologia que estavam cursando algum estágio em Psicologia foram os colaboradores da presente pesquisa. 0 número da amostra obtida foi pequeno (394) quando comparado ao contingente atual de estudantes de Psicologia em todo país. Apesar de a amostra não ser representativa, os dados obtidos forneceram informações importante sobre quem é este estudante, qual sua idade e onde estuda. Ademais, estudos de caracterização da população são essenciais para o planejamento de mudanças e aperfeiçoamento do funcionamento de instituições.

A expansão crescente dos cursos de ensino superior em Psicologia potencializa e prioriza a necessidade de estudos e debates acerca da formação do psicólogo. Como supervisionar e garantir a qualidade desta formação? Que psicólogo se quer formar: um generalista, um pesquisador, um tecnicista ou um transformador de realidades sociais? Como ampliar o leque de intervenções para diferentes contextos e populações e implementar tal práxis durante a formação do psicólogo?

Tais questões são de extrema relevância para o futuro da formação do psicólogo e para a sua 
inserção no mercado de trabalho. Pesquisas similares ao presente estudo são significativas e podem contribuir para ampliar o conhecimento sobre esta temática.

\section{Referências}

Achcar, R. (Org.) (1994). Psicólogo Brasileiro: práticas emergentes e desafios para a formação. São Paulo: Casa do Psicólogo.

Bastos, A. V. B. \& Achcar, R. (1994). Dinâmica profissional e formação do psicólogo: uma perspectiva de integração. In R. Achcar (Org.). Psicólogo Brasileiro: práticas emergentes e desafios para a formação (pp. 299-329). São Paulo: Casa do Psicólogo.

Bastos, A. V. B., Gondim, S. M. G. \& Rodrigues, A. C. A. (2010). Uma categoria profissional em expansão: quantos somos e onde estamos? In A. V. B. Bastos \& S. M. G. Gondim (Org.) O Trabalho do Psicólogo no Brasil (pp. 32-44). Porto Alegre: Artmed.

Brasil (1962). Lei n. 4.119, de 27 de agosto de 1962. Dispõe sobre os cursos de formação em Psicologia e regulamenta a profissão de psicólogo. Recuperado em 8 de janeiro de 2015, de http://www.planalto. gov.br/ccivil_03/leis/1950-1969/L4119.htm

Brasil (1996). Lei n. 9.394, de 20 de dezembro de 1996. Estabelece as diretrizes e bases da educação nacional. Recuperado em 8 de janeiro de 2015, de http:// www.planalto.gov.br/ccivil_03/leis/19394.htm

Brasil (2004). Ministério da Educação. Resolução no 8, de 7 de maio de 2004. Diretrizes Curriculares Nacionais para os cursos de graduação em Psicologia. Brasília: DF. Psicologia: Teoria e Pesquisa, 20(2), 205-208.

Conselho Federal de Psicologia. (1988). Quem é o psicólogo brasileiro? São Paulo: Edicon.

Conselho Federal de Psicologia (2001). Recuperado em 28 de abril de 2012 de http://www.pol.org.br/pol/ export/sites/default/pol/publicacoes/publicacoesDocumentos/Pesquisa_WHO.pdf
Instituto Nacional de Estudos e Pesquisas Educacionais Anísio Teixeira (2011). Ministério da Educação. Censo da Educação do Ensino Superior 2011. Recuperado em 28 de abril de 2012, de www.inep.gov.br

Lisboa, F. S. \& Barbosa, A. J. G. (2009). Formação em Psicologia no Brasil: um perfil dos cursos de graduação. Psicologia: Ciência e Profissão, 29(4), 718-737.

Löhr, S. S. \& Silvares, E. F. M. (2006). Clínica-Escola: integração da formação acadêmica com as necessidades da comunidade. In E. F. M. Silvares (Org.) Atendimento psicológico em clínicas-escola (pp. 11-22). Campinas: Alínea.

Nigan, T., Cameron, P. M. \& Leverette, J. S. (1997). Impasses in the Supervisory Process: a resident's perspective. American Journal of Psychotherapy, 51, 252-272.

Pereira, F.M. \& Pereira Neto, A. (2003). O psicólogo no Brasil: notas sobre seu processo de profissionalização. Psicologia em Estudo, Maringá, 8(2), 19-27.

Pessotti, I. (1988). Notas para uma história da psicologia brasileira. In Conselho Federal de Psicologia. Quem é o psicólogo brasileiro? (pp.17-31). São Paulo: Edicon.

Pfromm Netto, S. (2006). Aonde vai a Psicologia? Algumas considerações sobre o passado, o presente e o futuro da Psicologia como ciência, profissão e ensino. Boletim Academia Paulista de Psicologia, XXVI(3), 45-52.

Witter, G. P. \& Ferreira, A. A. (2005). Formação do Psicólogo hoje. In Conselho Federal de Psicologia. Psicólogo brasileiro: construção de novos espaços (pp. 15-39). Campinas: Alínea.

Witter, G. P., Gonçalves, C. L. C., Witter, C., Yukimitsu, M. T. C. P., \& Napolitano, J. R. (2005). In Conselho Federal de Psicologia. Psicólogo brasileiro: construção de novos espaços (pp. 40-67). Campinas: Alínea.

Yamamoto, O. H, Souza, J. A. J., Silva, N., \& Zanelli, J. C. (2010). A formação básica, pós-graduada e complementar do psicólogo no Brasil. In A. V. B. Bastos \& S. M. G. Gondim (Orgs.). O trabalho do psicólogo no Brasil (pp. 45-65). Porto Alegre: Artmed.

Psicol. Argum. 2014, 32(79), Supl. 2, 19-27 